\title{
Coarse Aggregate Size Effect on Non-linear and Uncertainty Mechanical Behaviors for Concrete
}

\author{
Ping Liu \\ China Ship Development and Design Center, The 701 Research Institute of CSIC, Wuhan, China
}

Email address:

flytoliuping@163.com

To cite this article:

Ping Liu. Coarse Aggregate Size Effect on Non-linear and Uncertainty Mechanical Behaviors for Concrete. Advances in Materials. Vol. 8, No. 3, 2019, pp. 100-107. doi: 10.11648/j.am.20190803.11

Received: April 9, 2019; Accepted: July 2, 2019; Published: July 19, 2019

\begin{abstract}
A series of uniaxial compression tests and simulations were conducted to evaluate the influences of single coarse aggregate (CA) size on the mechanical properties of concrete and their uncertainties. In this research, the specimens of pure mortar matrix and specimens with diameter $15 \mathrm{~mm}$ and $30 \mathrm{~mm}$ of single spherical steel aggregate were fabricated and tested by a material testing system. Based on experimental results, the mechanical parameters, including the elastic modulus, compressive strength, strain at the peak stress and absorbed strain energy were investigated. It was found that larger size of CA results in higher elastic modulus and compressive strength. Meanwhile, the strain at peak stress and absorbed strain energy of concrete are non-linear with the size of CA. And then, the mechanical properties were analyzed in the aspect of ITZ which is closely related to the size of CA. In addition, simulation results were presented to discuss the stress distribution of different size CA in specimen. In conclusion, the CA size has significant effect on the uncertainty of elastic modulus, strain at the peak stress and absorbed strain energy, but has little effect on the compressive strength. The findings from the current study will help gain the insights into the non-linear and uncertain mechanical behaviors of concrete.
\end{abstract}

Keywords: Concrete, Coarse Aggregate, Mechanical Property, Uncertainty, Non-linear

\section{Introduction}

Concrete, as one of the most widely used construction materials in the world, is a highly complex heterogeneous composite material with coarse aggregate (CA) embedded in mortar matrix and an interfacial transition zone (ITZ) in between. In most cases, ITZ behaves as a weak link separating the other two phases. The properties of the concrete are, thus, strongly related to the characteristics of $\mathrm{CA}$, the performance of mortar matrix, and the characteristics of ITZ [1]. Aggregates generally occupy 70 to $80 \%$ of the volume of concrete and among which around $45 \%$ are CAs. The influence of aggregate on mechanical properties of concrete, such as stress-strain curves, elastic modulus, compressive strength, fracture behavior, softening response, has been well studied [1-8]. It was found that both physical and mechanical properties of the aggregate, such as size, shape, strength and surface texture, have significant effects on the mechanical performance of concrete. The compressive strength of conventional concrete is mainly affected by the water-cement ratio $(\mathrm{w} / \mathrm{c})$. Other factors such as the cement content, sand concentration, aggregate characteristics are also known to affect concrete strength in varying degrees. The effect of $\mathrm{w} / \mathrm{c}$ ratio and cement paste volume on concrete behavior has been widely investigated for normal and highstrength concretes $[1,6]$.

On the other hand, there are growing interests in investigating the dependency of mechanical properties of concrete on the aggregate characteristics as it plays an important role for the properties of ITZ. The ITZ, as the weakest component, has important effect on the properties of concrete [9-15]. Numerous studies have been carried out to assess the effect of aggregates shape (rounded or irregular), surface texture (smooth or rough), and size on the microstructures and mechanical properties of ITZ [16-21]. In general, the ITZ bond can be strengthened with rough surface texture and irregular aggregate shape. Depending on the aggregate type and size, the bond properties of ITZ may also be strengthened or weakened by mediating the interaction between cement paste and aggregate [16-17]. However, no information on the non-linear mechanical properties and their uncertainty to the properties of CA/ITZ is available. 
It has been found that the thickness of transition zone is about 10 to $50 \mu \mathrm{m}$ with the porosity [18-20]. The characteristics of ITZ depend on the surface roughness, type, size of aggregate and the w/c ratio. The porosity of ITZ could lead to the uncertainty of interfacial bond stress transition between the aggregates and matrix. Moreover, the initial micro-cracks which may present in concrete even before application of any load could also contribute to the uncertainty of the properties [5]. The main causes of the preliminary micro-cracks are the differences of elasticity modulus and shrinkage between the aggregate and the matrix [22]. These initial micro-cracks randomly distribute in the concrete with different sizes, shapes and directions. The randomness of initial damages in ITZ indicates that the mechanical performance of concrete has uncertainty with respect to the microstructure of porosity and initial micro-cracks [23-25].

A series uniaxial compression tests were conducted in this study to investigate the effect of CA size on the mechanical properties and the uncertain behaviors of concrete. To achieve the objectives, the three-phase concrete was simplified by using a mortar matrix with a single relatively rigid, nonporous and smooth surface aggregate to highlight the effect of CA size. Single spherical steel aggregates with different sizes are adopted for different types of specimens to obtain the insights into the non-linear and uncertain mechanical behaviors of concrete.

\section{Experimental Procedures}

\subsection{Materials and Mix Proportions}

Ordinary Portland cement is used for the manufacture of concrete. The mortar matrix is prepared with natural river sands (maximum size $4 \mathrm{~mm}$ ) and tap water. The mix proportions of this specific mortar matrix are given in Table 1. Spherical steel balls with diameters of $15 \mathrm{~mm}$ and $30 \mathrm{~mm}$, which will be placed into the mortar matrix, are used to mimic CA in the same nominal size, as shown in Figure 1.

Table 1. Mix proportions of mortar matrix.

\begin{tabular}{ll}
\hline Ingredients & $\mathbf{k g} / \mathbf{m}^{\mathbf{3}}$ \\
\hline Sand & 1300 \\
Cement & 650 \\
Water & 210 \\
\hline
\end{tabular}

\subsection{Preparation of Specimens}

The cylindrical specimens with a diameter of $90 \mathrm{~mm}$ and a height of $45 \mathrm{~mm}$ are made by using PVC moulds, as shown in Figure 1. All specimens were covered with wet cloth under room temperature after casting. After 24 hours, the specimens were demoulded and put into incubator at temperature of $20.0^{\circ} \mathrm{C}$ and $90 \%$ of relative humidity until 28 days before testing. The compressive strength of mortar matrix prepared for tests is $49 \mathrm{MPa}$, which is obtained by the testing of cubic specimens with size of $70 \mathrm{~mm}$. In this research, the specimens of pure mortar matrix and specimens with diameter $15 \mathrm{~mm}$ and $30 \mathrm{~mm}$ of single spherical steel aggregate are prepared for comparative study. The single spherical steel aggregate is placed in the center of cylindrical mortar matrix, as shown in Figure 1. Both ends of the cylindrical specimens are polished with alumina to a surface roughness of less than $0.5 \mathrm{~mm}$. The specimens are specifically designed to investigate the effect of CA size with only one single spherical steel aggregate embedded in the center of the mortar matrix. Such design can eliminate the effects from other aggregate's characteristics, such as the shape, surface texture, mineral character, quantity and grading. Such experiments can relatively provide the insights into the dependency of concrete mechanical properties on the size of CA.

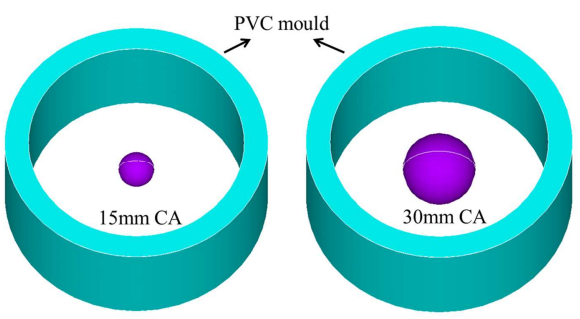

Figure 1. Spherical steel balls and PVC moulds.

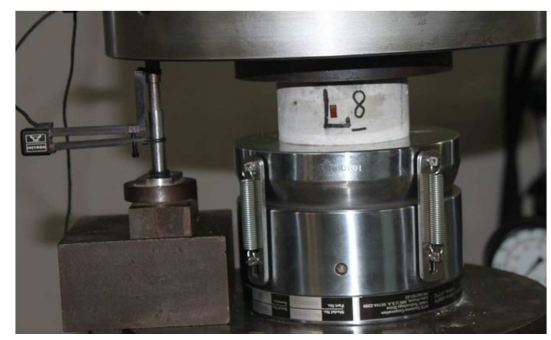

Figure 2. Uniaxial compression of a specimen.

\subsection{Testing Method}

The specimens were tested by a material testing system (MTS) with a maximum loading capacity of $2000 \mathrm{KN}$, as shown in Figure 2. A constant displacement rate of 0.2 $\mathrm{mm} / \mathrm{min}$ was applied for the uniaxial compression loading on the specimens to get the stress-strain relationship of the concrete. The axial compression and the vertical deformation were automatically collected by MTS and were validated by displacement sensor, in which the preloading is applied on the cylindrical specimen to eliminate the influence of contact.

\section{Experimental Results and Discussions}

\subsection{Experimental Results}

The stress-strain curve of each individual test and the averaged value are all shown in Figures 3 (a-c). In Figure 3, stress is obtained by applied force divided by cross sectional area of cylindrical specimen, and strain is obtained by axial displacement divided by height of specimen. The repetitive tests of 4,9 and 5 for specimens with diameters $0 \mathrm{~mm}, 15$ $\mathrm{mm}$ and $30 \mathrm{~mm}$ of CA were separately used to validate the reliability of experimental results. It should be noted that more tests were conducted for specimens with $15 \mathrm{~mm} \mathrm{CA}$, 
due to its larger variations of the stress-strain curves than those with diameters $0 \mathrm{~mm}$ and $30 \mathrm{~mm}$ of CA. Figure 3 (d) compares the mean curves of the stress-strain relationship obtained from different specimens. It can be found from Figure 3 that the non-linear relationship is observed between the stress and the strain, and the size of single spherical CA is sensitive to the stress-strain curves.
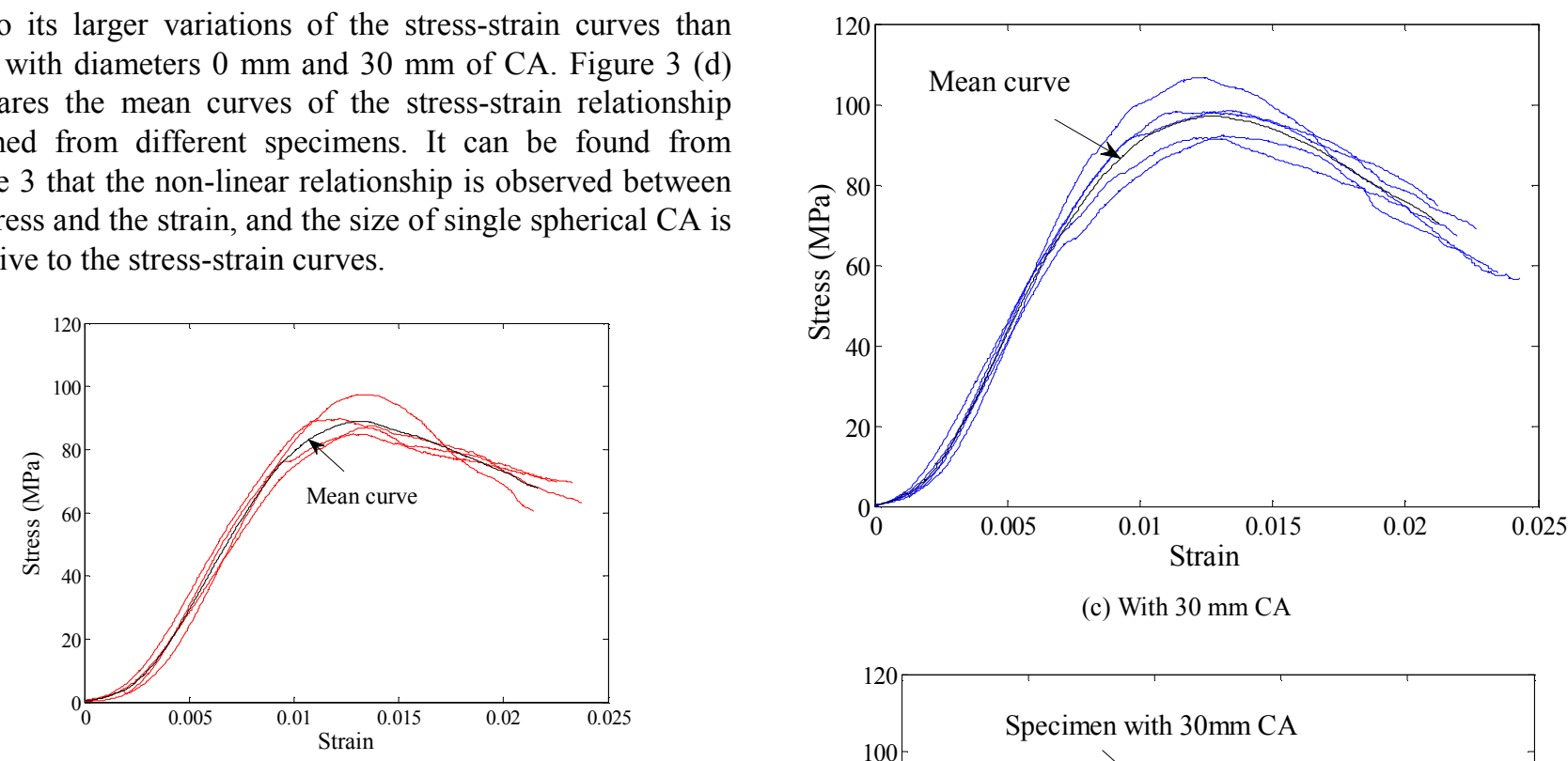

(c) With $30 \mathrm{~mm} \mathrm{CA}$

(a) Without CA

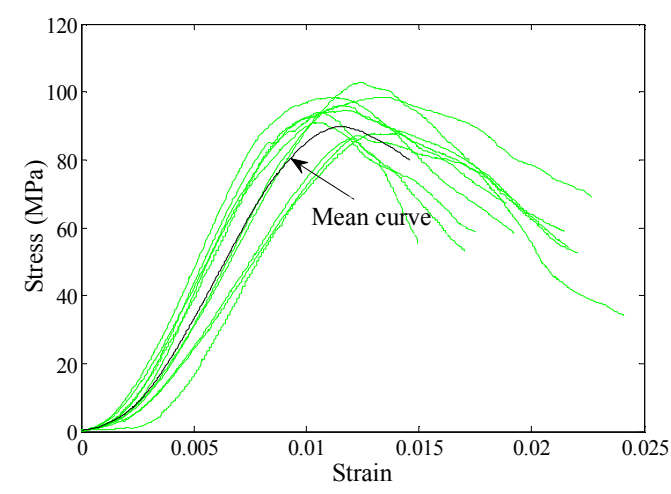

(b) With $15 \mathrm{~mm} \mathrm{CA}$

Table 2. Mechanical parameters of different specimens.

\begin{tabular}{|c|c|c|c|c|c|c|}
\hline \multirow{2}{*}{ Mechanical parameters } & \multicolumn{2}{|c|}{ Pure mortar matrix } & \multicolumn{2}{|c|}{ With 15 mm CA } & \multicolumn{2}{|c|}{ With 30 mm CA } \\
\hline & Mean value & Standard deviation & Mean value & Standard deviation & Mean value & Standard deviation \\
\hline Elastic modulus $(\mathrm{GPa})$ & 23.39 & 0.0087 & 24.88 & 0.0357 & 27.17 & 0.0174 \\
\hline Compressive strength (MPa) & 89.94 & 5.4205 & 93.14 & 5.5708 & 97.54 & 6.0829 \\
\hline Strain at the peak stress & 0.0130 & 0.0007 & 0.0117 & 0.0010 & 0.0125 & 0.0007 \\
\hline Absorbed strain energy (MJ) & 0.5916 & 0.0388 & 0.5216 & 0.0608 & 0.6703 & 0.0576 \\
\hline
\end{tabular}

Mechanical parameters of the concrete with different CA size are extracted from the stress-strain curves, including elastic modulus, compressive strength, strain at the peak stress and absorbed strain energy, as presented in Table 2. The elastic modulus is obtained by the secant slope of stressstrain curves at the $40 \%$ stress of the compressive strength. The strain energy is obtained by the integration of the stressstrain curves up to the peak stress, which denotes the absorbed strain energy of different types of the specimens before ultimate strength (damage) under uniaxial compression of concrete. The units of elastic modulus, compressive strength and strain energy are GPa, MPa and $\mathrm{MJ}$, respectively. The volume fraction of $\mathrm{CA}$ is $0,0.0062$ and 0.0494 for specimens of pure mortar matrix, $15 \mathrm{~mm} \mathrm{CA}$ and
$30 \mathrm{~mm} \mathrm{CA}$, separately. Figure 4 shows relationship between mean values of mechanical parameters and the volume fraction of CA. It can be observed that concrete with the larger volume fraction of CA shows higher elastic modulus and compressive strength when a single spherical steel CA placed in the center of the cylindrical specimen. There are monotonic relationship between elastic modulus, compressive strength and volume fraction of CA. However, the strain at the peak stress and the absorbed strain energy are not monotonic with the size of CA. Comparing to the specimens with $0 \mathrm{~mm}$ and $30 \mathrm{~mm} \mathrm{CA}$, their failure strains and corresponding strain energy of specimens with $15 \mathrm{~mm}$ $\mathrm{CA}$ are smaller. 


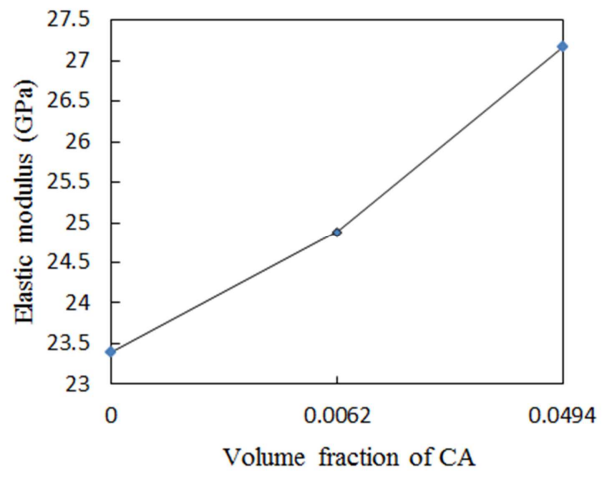

(a) Elastic modulus

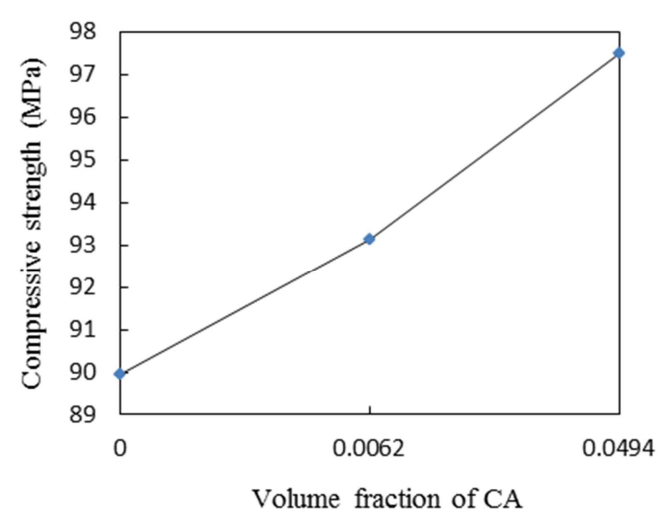

(b) Compressive strength

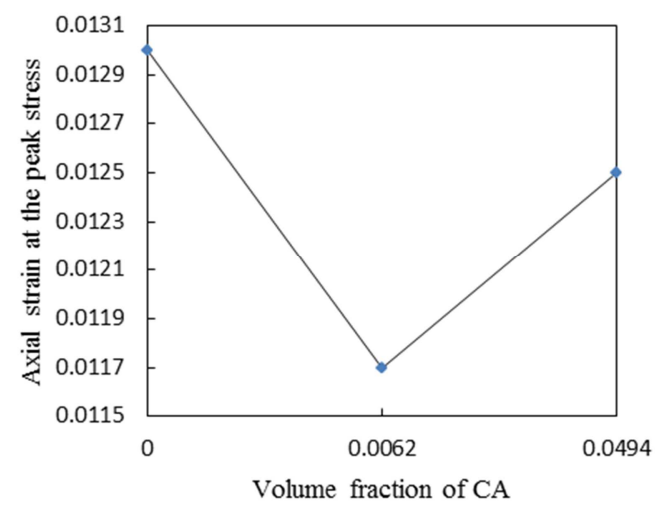

(c) Strain at the peak stress

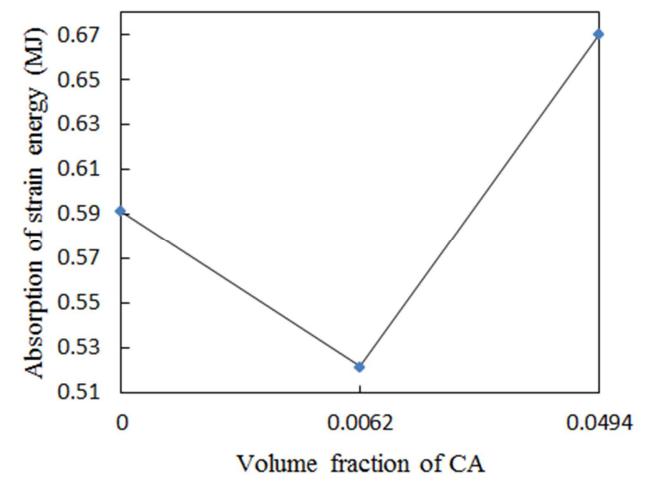

(d) Absorbed strain energy

Figure 4. Relationship between mean values of mechanical parameters and volume fraction of $C A$.

\subsection{Analysis of Mechanical Properties and Its Non-linear Performance}

The mechanical performance of concrete is integrated results of mortar matrix, CA and ITZ. In section 2, only effect of a single CA size on ITZ and subsequently on mechanical performance of concrete were studied under uniaxial compression. The specimens were prepared with the same mortar matrix, and the only variable is the size of CA which resulted in varied volume fractions of $\mathrm{CA}$ and varied ITZ between mortar matrix and CA.

Figure 4 (a) shows that the volume fraction of $\mathrm{CA}$ has a significant effect on the elastic modulus. According to the classical theory in mechanics composite material, this can be attributed to the significantly higher elastic modulus of spherical steel aggregate than the mortar matrix. The elastic modulus of concrete can thus increase with the volume fraction of CA. On the other hand, the bond strength of ITZ has stronger effects on the tensile stress of concrete but plays little role on the compressive stress [21]. Compressive stress of concrete will increase with elastic modulus at the same strain level. The compressive strength, therefore, is also increased with the volume fraction of CA, as shown in Figure 4 (b). Figure 4 (c) shows that strains at the peak stress (i.e. strain at compressive strength) of specimens with $15 \mathrm{~mm} \mathrm{CA}$ and $30 \mathrm{~mm} \mathrm{CA}$ are smaller than that of pure mortar matrix. Due to the ITZ constitutes the weakest link between CA and mortar matrix, under uniaxial compression, the lateral splitting tensile stresses can be created with the failure of ITZ. This is because the mortar matrix tends to flow around CA when interfacial force reaches to an ultimate load of ITZ, and the tensile strength is significantly smaller than the compressive strength for ITZ and mortar matrix [21].

In general, the larger size of $\mathrm{CA}$, the larger thickness of ITZ is formed [21]. And the larger thickness of ITZ, the larger amount of microcracks and voids are presented in the ITZ [18]. Thus the bond strength of ITZ may decrease with the increasing of CA size. An interesting phenomenon is that strain at the peak stress of specimens with $30 \mathrm{~mm}$ CA is larger than that of specimens with $15 \mathrm{~mm} \mathrm{CA}$, as shown in Figure 4 (c), where bond strength of specimens with $30 \mathrm{~mm}$ $\mathrm{CA}$ is smaller than that of specimens with $15 \mathrm{~mm}$ CA. The surface area of CA is $A=4 \pi r^{2}$ for a single spherical steel aggregate, where $r$ is radius of spherical CA. The larger size of CA, the larger surface area of ITZ can be obtained for specimens. The relationship between the ultimate load $F_{I}$ of interaction in ITZ and the radius $r$ of CA can be expressed by

$$
F_{I}=\propto\left[\sigma\left(\frac{1}{r}\right)\right] \propto[A(r)]
$$

where $\sigma\left(\frac{1}{r}\right)$ is the bond strength of ITZ, $A(r)$ is the surface area of ITZ, $\propto$ is the symbol that denotes the positive relationship. It indicates that the ultimate load is proportional to bond strength and surface area of ITZ. However, the bond strength $\sigma$ is monotonically decreasing with the size of CA, and the surface area $A$ is monotonically increasing with the diameter of CA. Figure 3 (d) shows that the ultimate load of 
failure for specimens with $15 \mathrm{~mm} \mathrm{CA}$ is smaller than that of specimens with $30 \mathrm{~mm} \mathrm{CA}$, where ultimate load is the applied force at the peak stress and can be obtained by stress multiply by cross sectional area of cylindrical specimen. The ultimate load should be proportional to the maximum force of interaction in ITZ, due to the ITZ constitutes the weakest link between CA and mortar matrix. The less ultimate load, the maximum force of interaction in ITZ will be smaller. Moreover, the surface area $A$ of $15 \mathrm{~mm} \mathrm{CA}$ is also smaller than that of $30 \mathrm{~mm} \mathrm{CA}$, the force of interaction in ITZ could be decreased when the surface area $A$ is decreased and the value of bond strength $\sigma$ is increased. Then, the smaller the maximum force of interaction in ITZ, the smaller strain at the peak stress will be obtained, as shown in Figure 4 (c). Herein, we should note that the strain at the peak stress is the engineering strain of specimens, which is not the true strain of ITZ under uniaxial compression. The non-monotonic relationship between interaction of ITZ and size of CA indicates that the failure of concrete can be significantly influenced by the size of CA. Furthermore, the smaller strain at the peak stress of specimens with $15 \mathrm{~mm} \mathrm{CA}$, the smaller absorbed strain energy is obtained compared to other types of specimens.

\subsection{Uncertain Behavior of Mechanical Parameters}

For the above-mentioned specimens, the single spherical steel CA used is a homogeneous medium, whose mechanical uncertainty is quite limited. Therefore, the uncertain behaviors of these specimens are mainly induced by the properties of mortar matrix and ITZ, whose microstructures are complex comparing to the medium of CA. The uncertain factors in mortar matrix are caused by the stochastic distribution of fine aggregates and pores structure. The uncertain properties of ITZ are results of the porosity and initial micro-cracks. The stress-strain curves in Figure 3 show that the uncertain behaviors of different specimens are affected by the size of CA and the level of uncertainty has non-linear response to the CA size. The area of uncertain interval of stress-strain curves in Figure 3 is $0.1736,0.4678$ and 0.1727 for specimens with CA diameters of $0 \mathrm{~mm}, 15$ $\mathrm{mm}$ and $30 \mathrm{~mm}$, separately, which is obtained by computing the area of uncertain interval up to the strain of 0.02 . The area of uncertain interval of specimens with $15 \mathrm{~mm} \mathrm{CA}$ is larger than those of $0 \mathrm{~mm}$ and $30 \mathrm{~mm} \mathrm{CA}$.

The uncertain behavior of concrete under uniaxial compression can be further summarized by using the mechanical parameters of elastic modulus, compressive strength, strain at the peak stress and absorbed strain energy. The uncertainty of these mechanical parameters is evaluated by the coefficient of variation, which is given by

$$
X=\sqrt{\operatorname{var}(x)} / E(x)
$$

and

$$
\sqrt{\operatorname{var}(x)}=\sqrt{\sum_{i=1}^{n}\left(x_{i}-E(x)\right)^{2}} /(n-1)
$$

where $\sqrt{\operatorname{var}(x)}$ is the standard sample variance, $E(x)$ is the mean value of mechanical parameters, $x_{i}$ and $n$ is the measured results and number of specimens for tests, respectively.

Figure 5 gives the coefficients of variation of elastic modulus, compressive strength, strain at the peak stress and absorbed strain energy. It can be seen that the CA size has a significant effect on the uncertainty of elastic modulus, strain at the peak stress and absorbed strain energy. The coefficients of variation of specimens with $15 \mathrm{~mm} \mathrm{CA}$ are remarkable larger than those of $0 \mathrm{~mm}$ and $30 \mathrm{~mm} \mathrm{CA}$, in which the uncertainty of single medium of mortar matrix is removed from the effect of ITZ between CA and mortar matrix. Moreover, Figure 6 shows the distribution of von Mises stresses obtained by simulations for specimens with 15 $\mathrm{mm}$ and $30 \mathrm{~mm} \mathrm{CA}$ under the same pressure. Where the material of mortar matrix is modeled by isotropic and homogeneous medium, the uncertain properties of mortar matrix and ITZ are not considered in Figure 6. Figure 6 is only used for discussion the stress distribution of different size CA in specimen. It can be seen that the von Mises stresses distributed in CA for specimen with $30 \mathrm{~mm}$ CA are larger than those of specimen with $15 \mathrm{~mm} \mathrm{CA}$. The ranges of von Mises stresses in $\mathrm{CA}$ are 454.67-570.21 $\mathrm{MPa}$ for specimen with $30 \mathrm{~mm} \mathrm{CA}$ and 384.20-424.93 $\mathrm{MPa}$ for specimen with $15 \mathrm{~mm} \mathrm{CA}$. The comparison of the stress distribution in 15 and $30 \mathrm{~mm} \mathrm{CA}$ indicates that, when the size of CA is larger, more strain energy can be absorbed by $\mathrm{CA}$ before the failure of specimens. Due to the homogeneity of the steel CA, the uncertain behaviors of specimens should decrease when most of the strain energy is absorbed by CA. Therefore, it can be concluded that the uncertainty of mechanical parameters, including elastic modulus, strain at the peak stress and absorbed strain energy, are increased with the size of CA at the beginning, which is facilitated by the uncertain properties of ITZ. After the uncertainty approaches to a maximum level, the uncertainty should decrease with the increase of the volume fraction of CA. Both the experimental results and the simulations indicate that the level of uncertain behaviors in concrete has a non-linear dependency on the CA size.

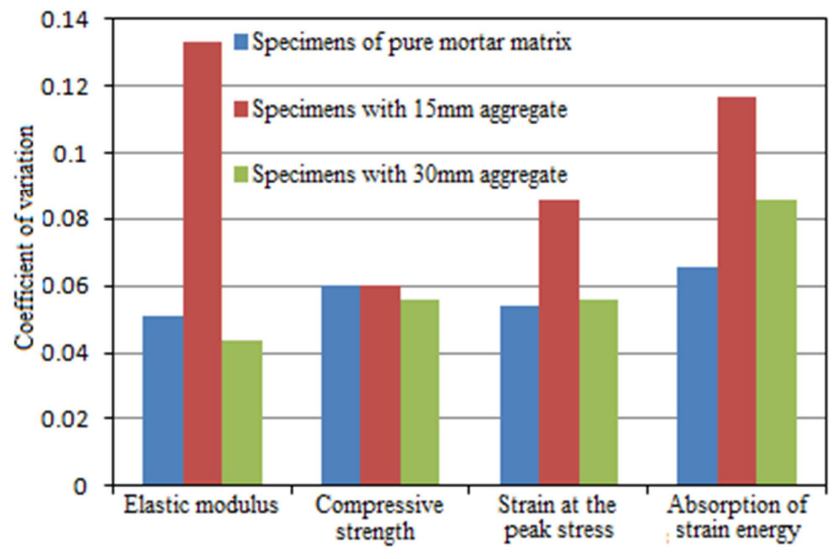

Figure 5. Coefficient of variation of mechanical parameters. 
On the other hand, it can also be found from Figure 5 that the CA size has little contribution to the uncertainty of compressive strength. As reported in reference [21], the intricate interaction of ITZ plays limited role on the compressive strength. Then the uncertain characteristic of
ITZ for specimens with different CA size also has little effect on the uncertain behavior of compressive strength. This means that compressive strength is a reliable mechanical parameter for the design of concrete structures by changing the concrete mix proportions.
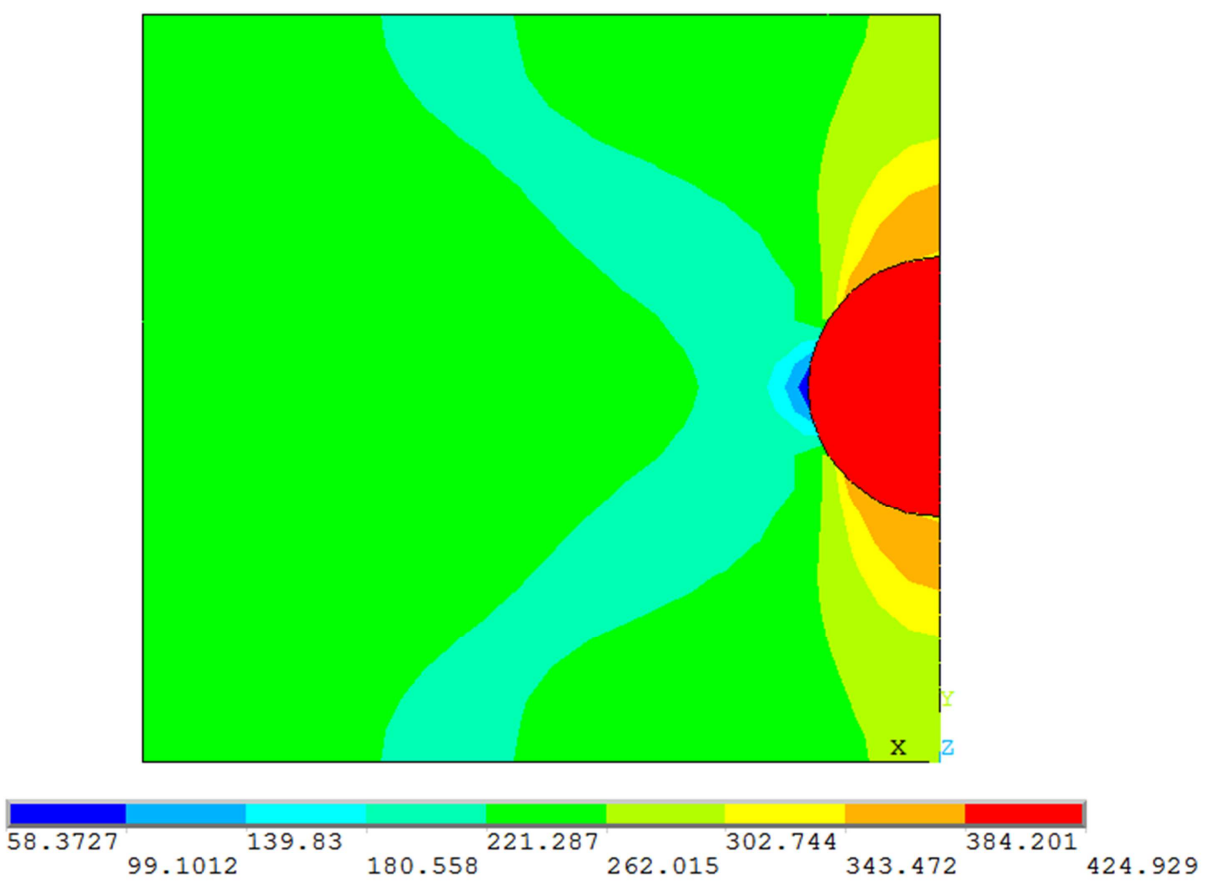

(a) With $15 \mathrm{~mm} \mathrm{CA}$

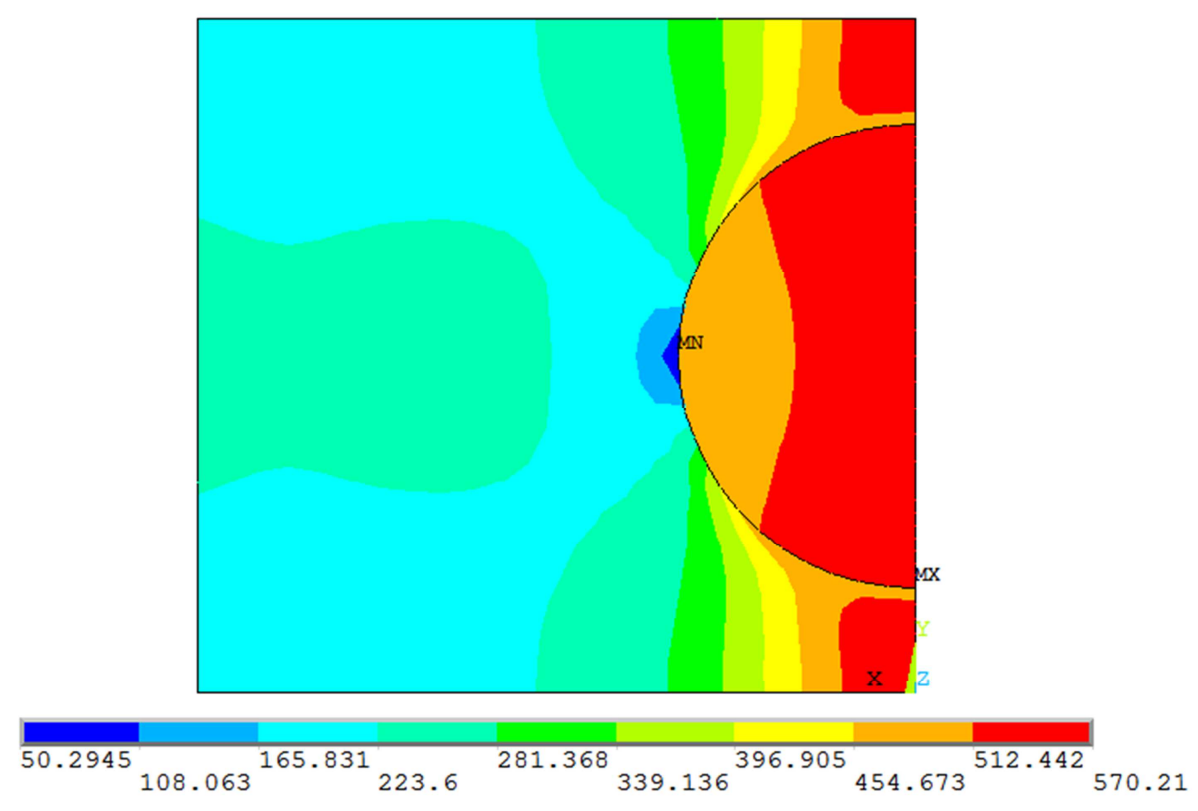

(b) With $30 \mathrm{~mm} \mathrm{CA}$

Figure 6. Distribution of von Mises stresses for specimens with different size of CA.

Figure 5 indicates that the CA size has significant effect on the uncertain behavior of strain at the peak stress but plays little role on the compressive strength, which is critical for the failure analysis of concrete material with specifically designed CA embedded. The compressive strength, in which the larger mean value can be obtained with a larger size of CA, cannot represent the significant effect of CA size on the uncertain behavior of mechanical parameters. The strain at the peak stress, in which the mean values of specimens with $15 \mathrm{~mm}$ aggregate are smaller than those of others, can be 
more appropriate for the analysis of failure problems of concrete comparing to compressive strength.

\section{Conclusions}

This study focuses on identifying the effect of CA size on non-linear mechanical parameters and its uncertain behavior of concrete under uniaxial compression loading. With the experimental study and numerical analysis, the following conclusions can be drawn.

(1) The mechanical properties of the concrete, including stress-strain curves, elastic modulus, compressive strength, strain at the peak stress and absorbed strain energy, are strongly dependent on the size of CA. There are monotonic relationship between the elastic modulus, compressive strength and the size of CA. However, the strain at the peak stress and the absorbed strain energy are not monotonic with the size of CA.

(2) The experimental results show that the CA size has significant effect on the uncertain behavior of elastic modulus, strain at the peak stress and absorbed strain energy, but has little effect on that of compressive strength. It indicates that compressive strength is a reliable mechanical parameter for the design of concrete structures.

(3) Strain at the peak stress of concrete could be significantly influenced by the lateral splitting tensile stresses in ITZ under uniaxial compression. And the strain at the peak stress is more sensitive to the failure of concrete comparing to compressive strength.

\section{Acknowledgements}

Financial supports from National Natural Science Foundation of China (11102065, 11272118) and Development Project of Hunan University for Young Teachers are gratefully acknowledged.

\section{References}

[1] Meddah, M. S., Zitouni, S. \& Belâabes, S. (2010). Effect of content and particle size distribution of coarse aggregate on the compressive strength of concrete. Construction and Building Materials 24, 505-512.

[2] Radovani, B. A. (1990). Grain size of adopted aggregate influence on strain-softening of concrete. Engineering Fracture Mechanics 35, 709-718.

[3] Tasdemir, C., Tasdemir, M. A., Lydon, F. D. \& Barr, B. I. G. (1996). Effects of silica fume and aggregate size on the brittleness of concrete. Cement and Concrete Research 26, 6368 .

[4] Su, R. K. L. \& Bei, C. (2008). The Effect of Coarse Aggregate Size on the Stress-strain Curves of Concrete under Uniaxial Compression. HKIE Transactions 15, 33-39.

[5] Chen, B. \& Liu, J. (2004). Effect of aggregate on the fracture behavior of high strength concrete. Construction and Building
Materials 18, 585-590.

[6] Vu, X. H., Daudeville, L. \& Malecot, Y. (2011). Effect of coarse aggregate size and cement paste volume on concrete behavior under high triaxial compression loading. Construction and Building Materials 25, 3941-3949.

[7] Sengul, O., Tasdemir, C. \& Tasdemir, M. A. (2002). Influence of aggregate type on mechanical behavior of normal-and highstrength concretes. ACI Materials Journal 99, 528-533.

[8] Zhou, F. P., Lydon, F. D. \& Barr, B. I. G. (1995). Effect of coarse aggregate on elastic modulus and compressive strength of high performance concrete. Cement and Concrete Research $25,177-186$.

[9] Elsharief, A., Cohen, M. D. \& Olek, J. (2003). Influence of aggregate size, water cement ratio and age on the microstructure of the interfacial transition zone. Cement and Concrete Research 33, 1837-1849.

[10] Ollivier, J. P., Maso, J. C. \& Bourdette, B. (1995). Interfacial transition zone in concrete. Advanced Cement Based Materials 2, 30-38.

[11] Liao, K.-Y., Chang, P.-K., Peng, Y.-N. \& Yang, C.-C. (2004). A study on characteristics of interfacial transition zone in concrete. Cement and Concrete Research 34, 977-989.

[12] Scrivener, K., Crumbie, A. \& Laugesen, P. (2004). The Interfacial Transition Zone (ITZ) Between Cement Paste and Aggregate in Concrete. Interface Science 12, 411-421.

[13] Lee, G. C. \& Choi, H. B. (2013). Study on interfacial transition zone properties of recycled aggregate by micro-hardness test. Construction and Building Materials 40, 455-460.

[14] Lee, K. M. \& Park, J. H. (2008). A numerical model for elastic modulus of concrete considering interfacial transition zone. Cement and Concrete Research 38, 396-402.

[15] Zhu, W. \& Bartos, P. J. M. (2000). Application of depthsensing microindentation testing to study of interfacial transition zone in reinforced concrete. Cement and Concrete Research 30, 1299-1304.

[16] Prokopski, G. \& Halbiniak, J. (2000). Interfacial transition zone in cementitious materials. Cement and Concrete Research 30, 579-583.

[17] Tasong, W. A., Lynsdale, C. J. \& Cripps, J. C. (1999). Aggregate-cement paste interface: Part I. Influence of aggregate geochemistry. Cement and Concrete Research 29, 1019-1025.

[18] Hu, J. \& Stroeven, P. (2004). Properties of the Interfacial Transition Zone in Model Concrete. Interface Science 12, 389397.

[19] Zheng, J. J., Li, C. Q. \& Zhou, X. Z. (2005). Characterization of microstructure of interfacial transition zone in concrete. ACI materials journal 102, 265-271.

[20] Yue, L. \& Shuguang, H. (2001). The microstructure of the interfacial transition zone between steel and cement paste. Cement and Concrete Research 31, 385-388.

[21] Akçaoğlu, T., Tokyay, M. \& Çelik, T. (2004). Effect of coarse aggregate size and matrix quality on ITZ and failure behavior of concrete under uniaxial compression. Cement and Concrete Composites 26, 633-638. 
[22] Igarashi, S.-i., Bentur, A. \& Kovler, K. (2000). Autogenous shrinkage and induced restraining stresses in high-strength concretes. Cement and Concrete Research 30, 1701-1707.

[23] Yang, I. H. (2007). Uncertainty and sensitivity analysis of time-dependent effects in concrete structures. Engineering Structures 29, 1366-1374.
[24] Golewski, G. L. (2018). An assessment of microcracks in the interfacial transition zone of durable concrete composites with fly ash additives. Composite Structures, 200, 515-520.

[25] Simon, K. M., \& Kishen, J. M. C. (2018). A multiscale model for post-peak softening response of concrete and the role of microcracks in the interfacial transition zone. Archive of Applied Mechanics, 88 (4), 1-15. 